\title{
A study on curved surface laser ablation using beam profile approach
}

\author{
Vivek Garg, Deepak Marla and \\ Suhas S. Joshi* \\ Department of Mechanical Engineering, \\ Indian Institute of Technology, \\ Bombay, Mumbai - 400076, India \\ Fax: +91-22-2572-6875 \\ Email: vivekgarg@iitb.ac.in \\ Email: deepakmarla@iitb.ac.in \\ Email: ssjoshi@iitb.ac.in \\ *Corresponding author
}

\begin{abstract}
Laser ablation process was used to generate micro-scale curved surfaces that find applications such as micro-lens and hydrophobic surfaces. Curved surface laser ablation to produce micro-scale features generally involves altering the spatial beam profiles using masks with varying spatial opacity. The surface profile obtained mainly depends on the altered laser beam profile. This work aimed at understanding the influence of laser beam profile on the generated surface in curved surface laser ablation. The study was based on a 2D finite element model of laser ablation that considered target heating, heat transfer and vaporisation on a poly methyl methacrylate (PMMA) target. Using this model, surface profile was predicted for various profiles of laser intensity and a qualitative analysis of curved surface profiles was carried out. The quantitative comparison of the ablation depths for uniform beam was used to validate the numerical model. The results of ablation depth were found to be in good agreement with that of the experiments.
\end{abstract}

Keywords: laser ablation; curved surface; micro-lens; finite element model and beam profile approach.

Reference to this paper should be made as follows: Garg, V., Marla, D. and Joshi, S.S. (2017) 'A study on curved surface laser ablation using beam profile approach', Int. J. Additive and Subtractive Materials Manufacturing, Vol. 1, No. 1, pp.42-56.

Biographical notes: Vivek Garg is a Research Scholar and currently working on focused ion beam nanofabrication, process modelling and optimisation at Indian Institute of Technology Bombay, India and Monash University, Australia. He completed his BTech in Mechanical Engineering from College of Engineering Roorkee, India in 2011. He joined the Mechanical Engineering Department at Indian Institute of Technology Bombay, India for his Master's. He also authored a paper in international conference on laser micromachining. His research interest is in the area of micro-nanofabrication.

Deepak Marla obtained his Bachelor of Technology and a $\mathrm{PhD}$ in the Department of Mechanical Engineering from the Indian Institute of Technology Bombay, India in 2007 and 2014, respectively. After obtaining his PhD, he worked as Post-Doctoral Research Associate at the University of Illinois at Urbana Champaign, Illinois, USA. He was awarded the H.C. Orsted 
post-doc-COFUND fellowship in 2015 and is currently working as a Post-Doctoral Fellow at the Technical University of Denmark since September 2015. His research lies in the area of advanced manufacturing processes.

Suhas S. Joshi is currently the Rahul Bajaj Chair Professor and Head in the Department of Mechanical Engineering at the Indian Institute of Technology Bombay, India. He obtained his $\mathrm{PhD}$ in 1999 from the same institute where he currently works. His research lies in the area of modelling of manufacturing processes using cutting tools and in the area of advanced machining processes at micro-scales such as laser machining, electric discharge machining, electrochemical machining, etc. He has authored more than 100 articles in international journals.

This paper is a revised and expanded version of a paper entitled 'Numerical modeling of excimer laser curved surface ablation' presented at International Conference on Micro-Manufacturing, Victoria, Canada, 25 March 2013.

\section{Introduction}

Laser micro machining by ablation has proved to be a useful technique in many fields of advanced manufacturing over the past few decades due to growing need of miniaturisation, high speed and its precise machining abilities. Laser ablation is also used to produce curved surfaces. Curved surfaces using laser ablation can be obtained in using two different approaches. The first approach involves altering the beam profile by using greyscale masks (Braun and Zimmer, 2002); the second approach makes use of varying number of pulses along the path to be profiled (Naessens et al., 2002). Micro-scale curved surfaces find critical applications such as hydrophobic micro-scale surfaces and micro-lens arrays.

Micro-lens have recently found applications in areas like electronics and telecommunication, 3D projection astronomy, light field photography, confocal microscopy, beam delivery systems, etc. (Borrelli, 2004; Song et al., 2013). Currently, a great deal of research interest exists in their fabrication technologies (Feidhlim and Sheridan, 2002). Electron beam lithography was used to produce PMMA micro-lens (Fujita et al., 1981). Lens arrays employing moulds of suitable shape by hot embossing were made (Shen et al., 2002). A number of techniques like using elastomeric moulds of PDMS (Shin et al., 2004), monomers shaped using a patterned hydrophobic layer and UV cured to form micro-lens arrays (Hartmann et al., 2000), an ink jet apparatus to form micro-lens array (Christopher, 2007), greyscale lithography (McKenna et al., 2010), etc. have been developed. However, most of these techniques involve tedious processes and are time consuming. Curved surface laser ablation technique based on beam path approach due to its simplicity and quickness is considered as a potential technique micro-lens manufacturing (Braun and Zimmer, 2002). The surface profile of the ablated surface using this technique mainly depends on the profile of the mask that is used to alter the spatial profile of laser beam intensity. The design of mask profile usually involves a tedious process of empirical study of the influence of mask profile on the surface profile using experiments. 
Clearly, there is a need to understand the influence of mask profile on the generated surface profile that can help in efficiently designing the mask. Therefore, the present study aims to enhance the fundamental understanding of the nature of curved surface ablation using profiled path approach. In this work, a finite element-based 2D-numerical model of laser ablation has been developed to study the generation of curved surfaces using this technique and to establish a correlation between the laser profile and the generated surface profile. The modelling involves consideration to various phenomena that occur in laser ablation viz. laser-target interaction, heat transfer and vaporisation. The modelling has been carried out for a single pulse on a PMMA target. Using this model, surface profile has been predicted for various profiles of laser intensity and a qualitative analysis of curved surface profiles is carried out. The quantitative comparison of the ablation depths for uniform beam is done to validate the numerical model.

The outline of the paper is as follows. A description of the curved surface ablation technique using laser ablation is presented in Section 2. Section 3 presents the numerical modelling approach that is used to simulate the curved surface laser ablation process. The experimental set-up used in this work is described in Section 4. In Section 5, the simulation and experimental results are discussed along with the model validation. Finally, the important conclusions of the work are summarised in Section 6.

\section{Curved surface laser ablation: process description}

There exist two different techniques using laser ablation to fabricate micro lenses or curved surfaces:

1 beam profile approach (Braun and Zimmer, 2002)

2 profiled path approach (Naessens et al., 2002).

These techniques are based on the idea of changing the laser intensity or laser fluence across the surface to be ablated to achieve the desired profile. Beam profile approach is based on the idea of systematically changing the beam profile in accordance with the desired lens profile using a mask. A typical excimer laser micromachining setup along with the mask is shown in Figure 1. Greyscale mask is placed in the path of a laser beam to modify the beam as shown in Figure 2.

Figure 1 Schematic of beam profile approach technique using a mask (see online version for colours)

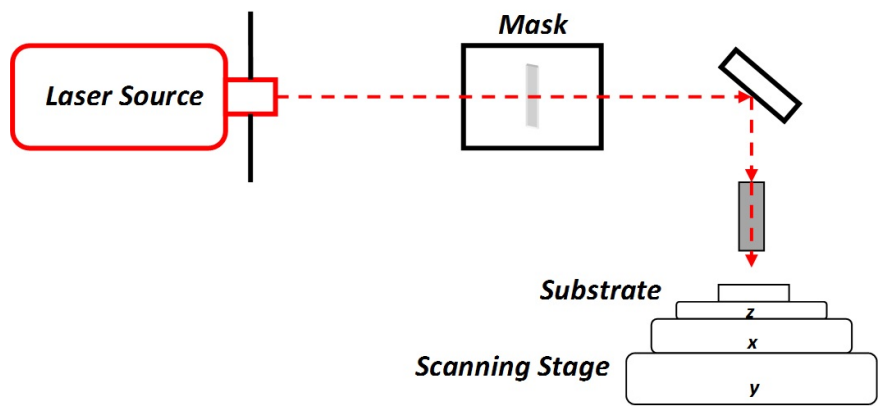


Figure 2 Modification of beam intensity profile using a mask (see online version for colours)

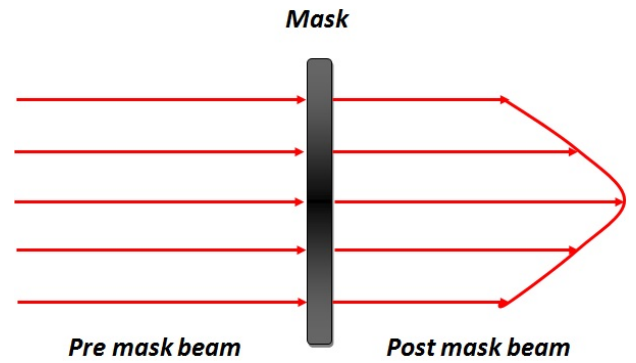

In typical ablation experiments, completely opaque optical masks, with cutout patterns are used to selectively alter the shape of the beam. These are binary masks where different areas of the beam are either transmitted or blocked. Although such masks can change the cross sectional shape of the beam, they cannot change the intensity profile of the transmitted beam. However, in order to control the shape of ablated crater, the intensity variation of the incident beam across its cross-section needs to be controlled. For this purpose a new category of optical masks with varying opacity, called greyscale masks are used instead of the traditional binary masks. In the greyscale mask ablation technique, an optical mask with varying opacity is used to alter the spatial intensity profile of the incident laser beam. Figure 3 shows typical masks of radially varying opacity used for fabrication of axisymmetric lenses. The spatial intensity profile of the beam coming out of the masks is no longer constant over the cross-section and such a modified beam creates a unique ablated surface profile. The shape of the ablated material is governed by the opacity variation of the greyscale optical mask used. Braun and Zimmer (2002) first discussed the use of greyscale masks for laser ablation to alter the beam profile. However, the technique was not explored in detail from fabrication point of view and the shape control of fabricated profiles was not investigated. Hayden (2003) also made greyscale masks using a computer algorithm. However, using these masks at different laser fluences resulted in significant distortion of the fabricated structures.

Figure 3 Greyscale optical mask with radially varying opacity
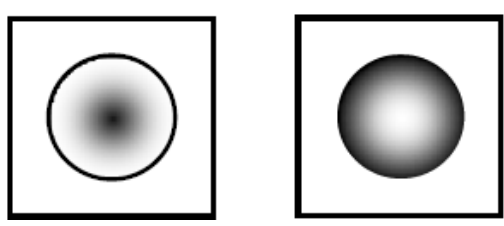

As the laser radiation passes through the mask, the greyscale mask acts as a translucent material reflecting a part of the radiation while transmitting the rest. Due to varying opacity of the greyscale mask, its intensity along the cross-section gets altered as shown in Figure 2.

Under the application of such a modified beam, the laser ablation produces a curved surface. The desired profile of the ablated surface can be obtained by selectively choosing the opacity profile of the greyscale mask. The intensity of the modified beam can be expressed as a function of the opacity function of the greyscale mask as: 


$$
I(x, y)=I_{L}\left(1-\rho_{o}(x, y)\right)
$$

where $I_{L}$ is the intensity of the original laser beam and $\rho_{o}(x, y)$ is the opacity function of the greyscale mask. The opacity function represents the reflectivity of the greyscale mask.

On the other hand, the technique based on profiled path approach uses a varying number of pulses along the workpiece to generate the desired profile. Unlike the earlier discussed technique, which uses varying intensity pulse focussed on a particular area, the intensity profile of the beam in this technique is unchanged, but uses varying number of pulses along the area to be profiled. As the ablation depth also depends on the number of pulses, craters or profiled surfaces can be fabricated by varying the number of pulses along the machining area.

The approach uses a simple circular hole as beam aperture for the excimer laser and the translation stage carrying the polymer substrate, capable of making circular movements. All laser related parameters like the pulse energy and the pulse frequency of the laser remain invariant during the process. While the excimer laser fires the pulses, the translation stage makes subsequent circular concentric scans with different radii and speeds. By careful choice of both radius and number of holes per circle, an arbitrary desired surface shape with circular symmetry can be obtained. Both convex and concave micro-lens can be obtained employing this approach.

However, the profiled path approach involves a tedious process and cannot be applied at micro-scales. In comparison, beam profile approach is both simple and effective at microscales. Therefore, the present study focuses on the beam profile approach.

\section{Model description}

\subsection{Laser ablation model}

Laser ablation involves removal of material due to heating that leads to melting and vaporisation of the target. When the target material is irradiated with laser energy, a certain fraction of it gets reflected, while the rest of the energy is absorbed by the target that causes its heating. In polymers, the absorbed radiation may cause photo-thermal or photo-chemical decomposition. Usually, the material removal is governed by the interplay of both these mechanisms. While vaporisation may occur at almost all laser fluences, bond breaking due to photo-chemical mechanism occurs only beyond a threshold fluence $\left(\approx 1.5 \mathrm{~J} / \mathrm{cm}^{2}\right.$ ) (Lazare and Granier, 1989). Due to the complexity in the photo-chemical mode ablation, in this work only photo-thermal mode of ablation is considered. In order for the above assumption to be valid, the operating laser fluence is considered to be very low in the range of $1-2 \mathrm{~J} / \mathrm{cm}^{2}$.

The heat transfer analysis of heating of the target due to laser radiation can be described using the Fourier heat conduction equation (Marla et al., 2011). The absorbed energy can be treated as a heat source term. The equation is given by,

$$
\rho c_{p} \frac{\partial T}{\partial t}=\frac{\partial}{\partial z}\left(K \frac{\partial T}{\partial z}\right)+Q
$$

where $\rho$ is the density, $c_{p}$ is the specific heat, $T$ is the temperature, $t$ is time, $K$ is the thermal conductivity and $Q$ is the laser heat source given by, 


$$
\begin{aligned}
& Q=(1-R) \alpha I \\
& \frac{d I}{d z}=-\alpha I
\end{aligned}
$$

where $R$ is the reflectivity, $\alpha$ is the absorption coefficient and $I$ is the laser intensity along the $z$ direction. The laser intensity varies along its cross-section and also with time. The surface profile generated by laser ablation is mainly dependent on the variation of laser intensity profile along its cross-section. The systematic modification of the intensity profile can be used to fabricate a variety of curved surfaces.

\subsection{Numerical modelling approach}

A finite element numerical model has been developed to simulate and study the curved surface ablation methodology using beam profile approach. Commercial software ANSYS 13 is used in the simulation. The model is based on a $2 \mathrm{D}$ heat conduction equation. Thermal analysis is considered in the simulations to estimate the temperature profile of the target and material removal (element deletion) based on the normal boiling temperature. Considering incremental time step of 1 ns over a pulse duration time of 20 $\mathrm{ns}$, this time dependent problem is solved sequentially. The target is represented by a mesh of finite elements and initial temperature of target is taken as $300 \mathrm{~K}$. Ablation is assumed to occur, when the temperature of an element is higher than the boiling point temperature $\left(\mathrm{T}_{b}\right)$ at the end of a particular sub-step. The output of the last time step is taken as input to the next time step. Phase change effects are taken into account by considering the latent heat of vaporisation $(L)$ in the model. A summary of the modelling approach is schematically represented in Figure 4.

Figure 4 Schematic of the numerical modelling approach carried out in the present work

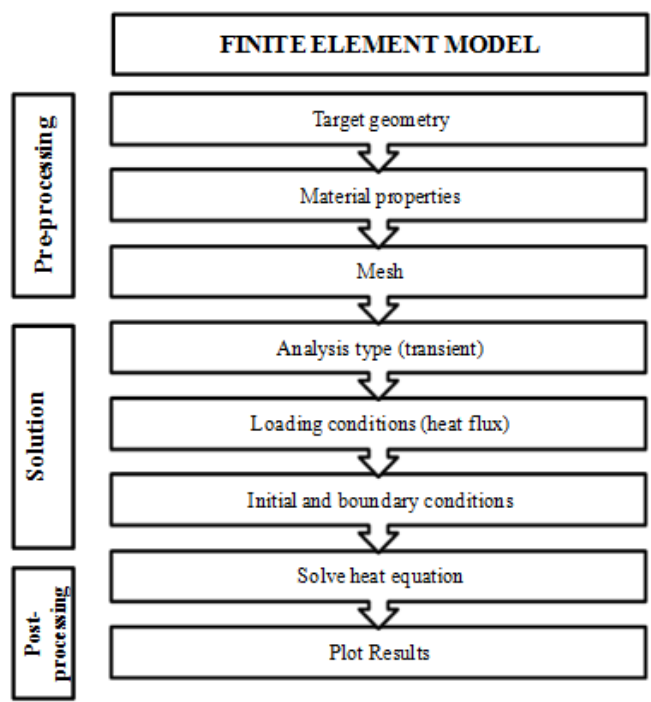




\subsection{Target geometry and material properties}

The target geometry used for simulation is shown in Figure 5. The target is considered to be rectangular in shape with dimension of $18 \mu \mathrm{m} \times 6 \mu \mathrm{m}$. Only half of the target is simulated because of the axial symmetry of the problem. Moreover, only part of the simulated region is irradiated in order to account for the lateral heat losses to the non-irradiated part of the sample. The size of each element is $5 \mathrm{~nm} \times 5 \mathrm{~nm}$. The element used for the analysis is PLANE 55. The element has four nodes with single degree of freedom at each node that can be applied for 2D, axisymmetric, steady state or transient thermal analysis. The thermophysical properties of PMMA (Agari et al., 1997) used in the simulation are listed in Table 1. The thermo-physical properties used in the simulation are temperature dependent as the thermal properties of PMMA changes significantly with temperature.

Figure 5 Schematic of the target geometry along with the incident laser beam and boundary conditions

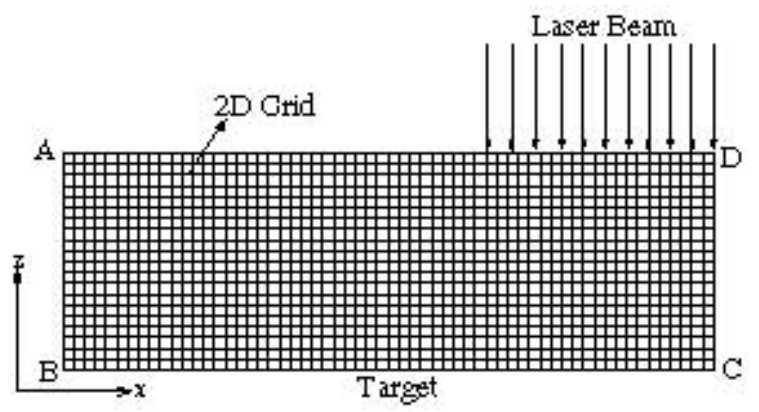

Table 1 Material properties of PMMA used in the simulation

\begin{tabular}{lccc}
\hline Temperature $(K)$ & Density $\left(\mathrm{kg} / \mathrm{m}^{3}\right)$ & $\begin{array}{c}\text { Thermal conductivity } \\
(\mathrm{W} / \mathrm{m}-\mathrm{K})\end{array}$ & Specific heat $(\mathrm{J} / \mathrm{Kg}-\mathrm{K})$ \\
\hline 300 & 1,135 & 0.29 & 1,450 \\
350 & 1,121 & 0.26 & 1,747 \\
400 & 1,107 & 0.23 & 2,044 \\
450 & 1,091 & 0.20 & 2,341 \\
500 & 1,079 & 0.17 & 2,638 \\
550 & 1,065 & 0.13 & 2,934 \\
600 & 1,051 & 0.10 & 3,230 \\
\hline
\end{tabular}

Source: Agari et al. (1997)

\subsection{Heat flux and boundary conditions}

The laser radiation is treated as heat flux and is applied on the irradiated surface as shown in Figure 5. The incident heat flux is taken as the pulse energy and the intensity is considered to be uniform with time. The boundary conditions considered in the model are given by, 


$$
\begin{aligned}
& \left.T(x, t)\right|_{z=0}=T_{0} \quad \text { (Along boundary BC in Figure 5) } \\
& \left.T(z, t)\right|_{x=0}=T_{0} \quad \text { (Along boundary AB in Figure 5) }
\end{aligned}
$$

The above two conditions are under the assumption that $\mathrm{AB}$ and $\mathrm{BC}$ in Figure 5 are far away and are at the same temperature. In addition,

$$
-\left.k(T) \frac{\partial T}{\partial x}\right|_{x=18 \mu m}=T_{0} \quad \text { (Along boundary AB in Figure 5). }
$$

Initially, the temperature of the body is equal to ambient temperature,

$$
T(z, 0)=T_{0}
$$

\subsection{Element removal technique}

The temperatures of the elements are calculated at the end of each sub-step. If the element temperature exceeds the melting temperature, ablation is assumed to occur and the elements above melting temperature have to be removed. Material removal in ANSYS is achieved through element birth and death technique, using the 'ekill' (command in ANSYS) to deactivate the elements. The element kill approach accounts for the vaporisation of PMMA. Also, the model assumes the polymer decomposition occurs at the boiling temperature. All the properties associated with the deactivated elements are set to zero, such as elements loads, specific heat, thermal conductivity, etc. Ablation position is easily identified by killed elements and their position. The shape and profile of the ablated surface can be obtained using this technique along with the temperature profile.

\subsection{Curved surface ablation modelling}

As mentioned earlier in Section 2, the curved surface ablation is achieved using non-uniform laser fluence. Instead of applying a uniform heat flux, spatially varying heat flux along the irradiated surface is used to simulate the curved surface ablation as shown in Figure 6. The profile of the laser radiation after passing through the grey scale mask is directly dependent on its opacity function. Therefore, the desired intensity profile can be obtained by choosing the opacity function of the greyscale mask.

Figure 6 Schematic of the target geometry along with the incident laser beam in beam profile approach (see online version for colours)

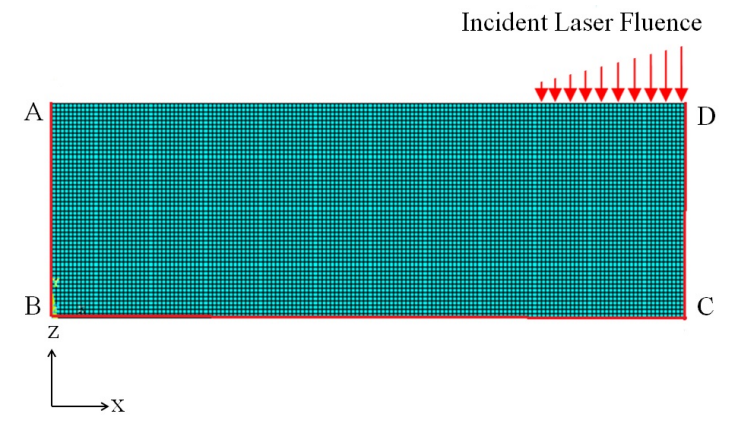


As there exists no direct correlation between the laser intensity profile and the ablated surface profile, a number of laser intensity profiles are considered in the simulation to study and establish a correlation between the two. Different profiles of laser intensity are considered in the model keeping the laser fluence constant. The profiles include:

1 top-hat (uniform beam)

2 exponential

3 Gaussian

4 ramp

5 parabolic.

\section{Experimental set-up}

Figure 7 shows the set-up used in the experiments. A Lamda Physik, $248 \mathrm{~nm} \mathrm{KrF}$ excimer laser setup equipped with a MicroLAS beam projection system has been used. A beam delivery system was used to project the laser beam radiated by the source onto the substrate. The beam delivery setup employs a homogeniser 2D array which makes the intensity of the beam constant over its cross-section. An optical mask with varying opacity is placed along the beam path (Figure 7) to alter the intensity profile of the laser beam. The laser beam passes through the optical mask and is then projected onto the workpiece placed on an XYZ-stage using projection lens. UV transparent quartz is used as a mask material, however, its life expectancy is low. The mask is damaged due to very high fluence of excimer laser and can be used for limited number of experiments. The mask is composed of diffractive sub areas (Braun and Zimmer, 2002). The greyscale mask allows defined amount of laser to pass based on its design and controls the beam profile. The technical specifications of the experimental ablation setup are presented in Table 2.

Figure 7 Photographic view of the experimental set-up (see online version for colours)

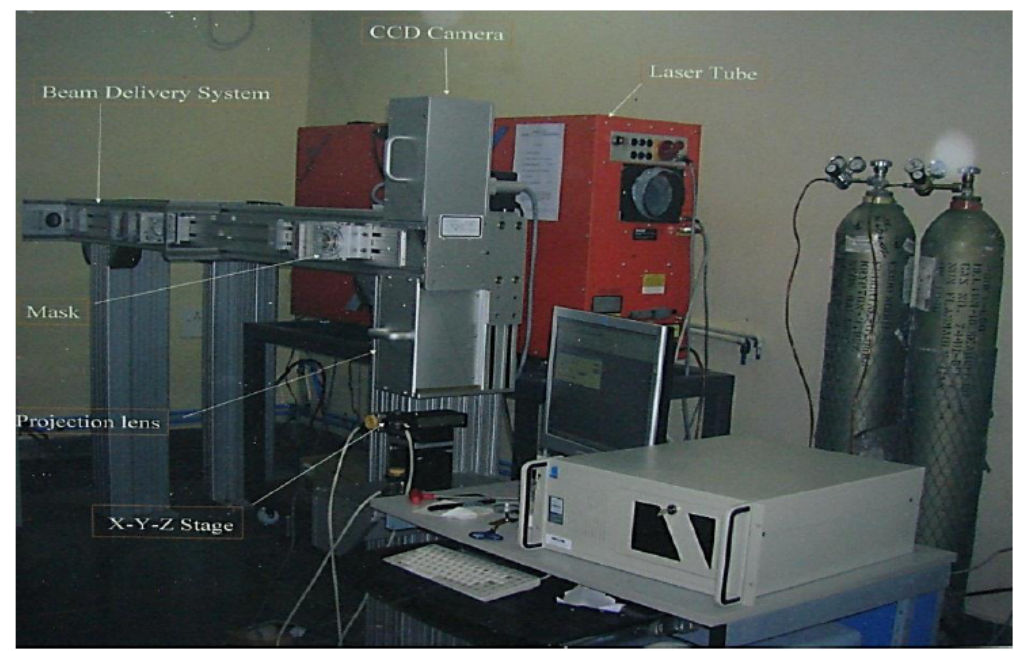


Table 2 Technical specifications of experimental setup

\begin{tabular}{lc}
\hline Wavelength & $248 \mathrm{~nm}(\mathrm{KrF})$ \\
Max. energy & $650 \mathrm{~mJ}$ \\
Pulse duration & $20 \mathrm{~ns}$ \\
Repetition rate (frequency) & $1-50 \mathrm{~Hz}$ \\
Beam size & $15 \mathrm{~mm} \times 15 \mathrm{~mm}$ \\
XYZ stage accuracy & $1 \mu \mathrm{m}$ \\
Resolution & 3 micron feature \\
\hline
\end{tabular}

\section{Results and discussion}

\subsection{Simulation of single pulse ablation}

In the initial analysis, single pulse simulations for un-modified beam (without mask) are carried out. The target surface is heated with single pulse of flat spatial profile and with laser fluence varying in the range from $1 \mathrm{~J} / \mathrm{cm}^{2}$ to $2.5 \mathrm{~J} / \mathrm{cm}^{2}$. Figure 8 (a) to Figure $8(\mathrm{~d})$ shows the simulation results for ablation depths for single pulse measured. Boiling point of PMMA is $327 \mathrm{C}$. The element birth and death process is employed to simulate material removal. The total pulse duration of $20 \mathrm{~ns}$ is divided into 20 sub-steps and after each sub-step all the elements with temperatures exceeding the boiling temperature are deleted. Figure 8(a) to Figure 8(d) shows the simulated profiles of ablation for the unchanged beam with same diameter at four different laser fluences for a single pulse. The four profiles in Figure 8(a) to Figure 8(d) clearly indicate that for an unmodified, uniform beam along the cross-section, the profile of the ablated surface is nearly flat except at the edges of the beam cross-section. The profile at the edge of beam cross-section is circular because of the heat conduction in the lateral (perpendicular to the depth) direction.

Figure 8 Simulated target profiles for laser fluence of, (a) $1 \mathrm{~J} / \mathrm{cm}^{2}$ (b) $1.5 \mathrm{~J} / \mathrm{cm}^{2}$ (c) $2 \mathrm{~J} / \mathrm{cm}^{2}$ (d) $2.5 \mathrm{~J} / \mathrm{cm}^{2}$ (see online version for colours)



(a)
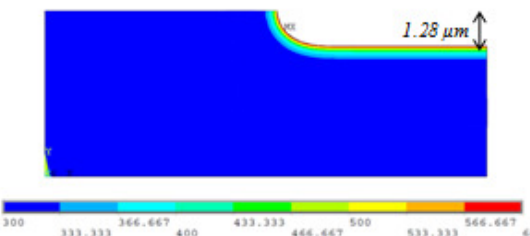

(c)

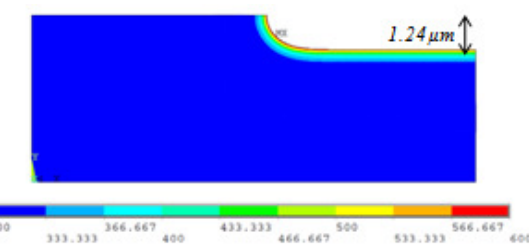

(b)

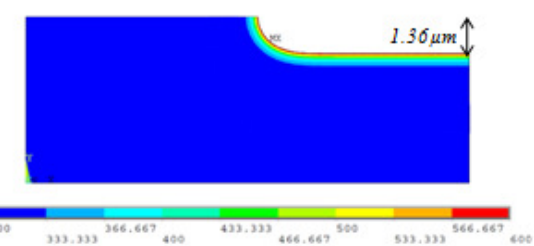

(d) 


\subsection{Model validation}

Experiments on PMMA substrate were carried out without optical mask at four different values of laser fluence of $1 \mathrm{~J} / \mathrm{cm}^{2}, 1.5 \mathrm{~J} / \mathrm{cm}^{2}, 2 \mathrm{~J} / \mathrm{cm}^{2}$ and $2.5 \mathrm{~J} / \mathrm{cm}^{2}$. The aim of the experiments was to calculate the ablated depth per pulse of the incident radiation in order to validate the simulation results. For ease of depth measurement, multiple pulses were fired and the total ablated depth was measured. Each experiment was carried out at pulse frequency $\mathrm{f}=10 \mathrm{~Hz}$ and the pulses were fired for a time of $\mathrm{t}=40 \mathrm{~s}$. Based on the experiments, an average value of ablation depth was measured. A white light interferometer (WYKO NT 1100) was used to measure the ablation depth. It is an optical profiler which provides three dimensional surface profile measurements without contact. The VSI mode based on vertical scanning interferrometry was used, which is dedicated to topography measurements upto $1 \mathrm{~mm}$.

Figure 9 Comparison of simulated results with that of experiments (see online version for colours)

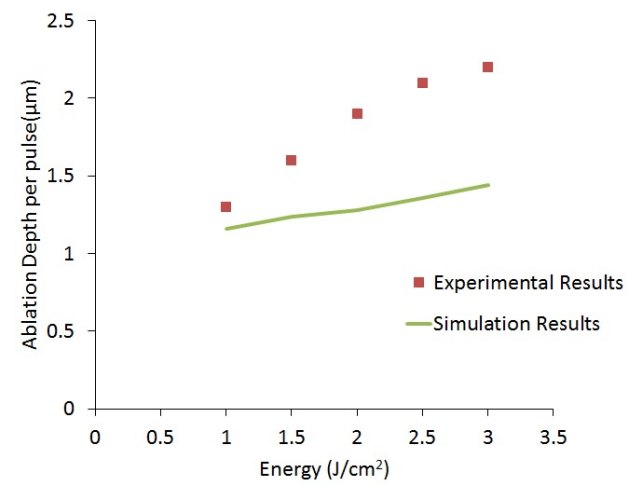

Figure 9 shows a comparison of simulated results with that of the experiments. The predicted values are in excellent agreement at a laser fluence of $1 \mathrm{~J} / \mathrm{cm}^{2}$. However, the simulation results under-predict the ablation depth at all other higher values of laser fluence. The discrepancy between the results increases with increasing laser fluence. This is mainly because the numerical model accounts only for the photothermal mechanism, while ignoring the photochemical mechanism and plasma recoil pressure of laser ablation. The photochemical mode of laser ablation involves material removal due to bond breaking as a result of photon absorption. However, it is to be noted that the photochemical mechanism initiates only beyond a certain threshold fluence. Below the threshold fluence, material removal due to photochemical breakdown is negligible, while it exponentially increases with an increase in fluence beyond the threshold fluence. Also, the model does not account for the effect of recoil pressure generated by PMMA vapor, which is significant at high laser fluences due to increased vaporisation. Recoil pressure generated by PMMA vapor is low at low laser fluences when the vaporisation is very low. So, the effect of recoil pressure can be ignored as the operating laser fluence is considered to be very low in the range of $1-2 \mathrm{~J} / \mathrm{cm}^{2}$. It is evident from the data in Figure 9 that the influence of photochemical model of ablation and recoil pressure is negligible at low laser fluence of $1 \mathrm{~J} / \mathrm{cm}^{2}$, which is mainly the reason for the excellent agreement between the simulated and the experimental results. The discrepancy in the 
results at higher fluences can be attributed to the photochemical model of ablation that becomes dominant at and above laser fluence of $1.5 \mathrm{~J} / \mathrm{cm}^{2}$ and effect of recoil pressure.

\subsection{Simulations on curved surface ablation}

In order to study the influence of laser intensity profile on the ablated surface profile, simulations are carried out for a number of laser beam profiles and the results of the ablated surface profile are studied. Curved surface simulations are carried out at low laser fluences due to the excellent results by the developed model at these values. Simulations based on the curved surface ablation model developed in the previous section are run with different beam profiles. Only the laser beam intensity profile is modified to different profiles, keeping all the conditions, i.e., laser fluence, pulse time and target material unchanged. The plots of variation of laser intensity across the beam cross-section for various profiles considered are shown in Figure 10. The profiles represent the variation of intensity for half the target considered for simulation in this analysis. The beam diameter has been kept same for all simulations. The plots are non-dimensionalised on both the axes. On the y-axis is plotted the intensity profile, non-dimensionalised with the intensity of top-hat (uniform beam). While the x-axis represents width of the target non-dimensionalised with half the width of the beam. The edge of the beam is taken as ' 1 ' while the origin is fixed at the centre of the beam.

Figure 10 Beam profiles considered in the model (see online version for colours)

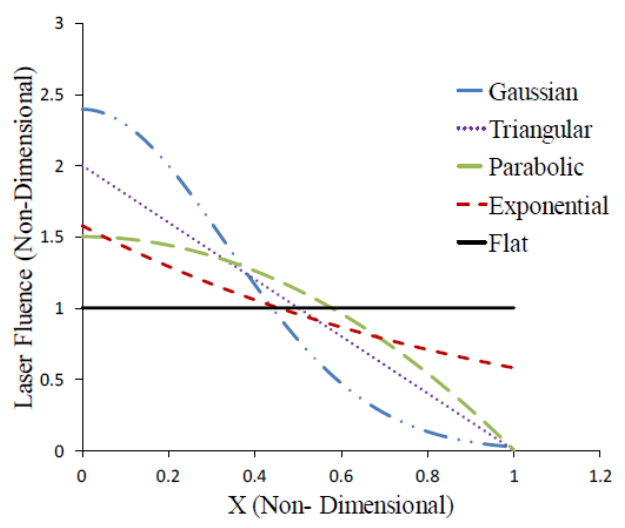

The simulation results of laser ablation for the above mentioned laser intensity profiles for a fluence of $1 \mathrm{~J} / \mathrm{cm}^{2}$ are shown in Figure 11(a) to Figure 11(d):

a exponential: $I_{0} e^{-x}$

b parabolic: $I_{0}\left(1-x^{2}\right)$

c ramp: $I_{0}(1-x)$

d Gaussian: $I_{0} e^{\left(-0.5\left(\frac{x}{\sigma}\right)^{2}\right)}$ (with beam width $\left.=3 \sigma\right)$. 
Figure 11 Simulated target profiles for curved surface ablation with various profiles, (a) uniform (b) Gaussian (c) parabolic (d) exponential (see online version for colours)

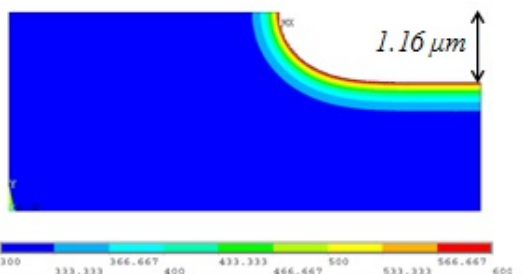

(a)

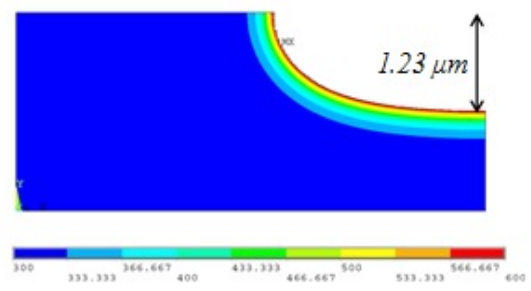

(c)



(b)

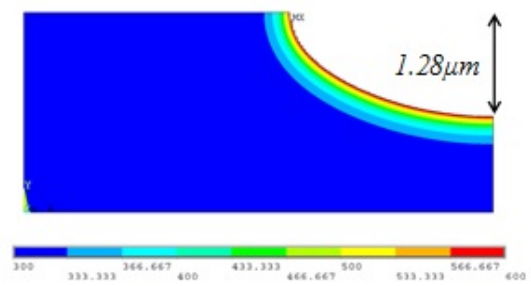

(d)

The plots clearly indicate the variation of surface profile for different profiles of laser intensities, although the ablation depths obtained are nearly the same. It is observed that the radius of curvature is minimum for a Gaussian profile, while maximum for uniform beam. It means that, the curvature of the surface obtained is much better for a Gaussian profile than all other profiles. This is mainly due to the steep variation in the intensity corresponding to the Gaussian profile, than compared with all other profiles. The nature of profile for a uniform beam is nearly flat. The crater width obtained is same in all simulation results due to the same beam diameter for each profile. In addition to ablation, polymers undergo glass-transition, resulting in a heat affected zone for the region above the glass transition temperature. The heat affected zone is nearly the same for different beam profiles (Figure 11), which is about $200 \mathrm{~nm}$ approximately. This suggests that the heat affected zone is independent of the beam profile. However, the curvature of the heat affected zone is similar to that of the ablated surface profile.

In another set of simulations, four different exponential profiles of the form $e^{(-k x)}$ for $k=1$ to 4 are considered keeping pulse energy and pulse duration same. Figure 12 shows plots of exponential profiles for different values of $k$ considered here. The simulation results for these four sets of laser intensity profiles are shown in Figure 13. Even in this case, it can be seen that the radius of curvature is different for different profiles with variations in the ablation depth at the centre of the target (right edge is the centre of the target as only half of the target is simulated). This suggests that the laser beam intensity profile decides the nature of the surface profile. Therefore, the numerical simulations can be used as an effective tool to generate curved surfaces with required curvature. 
Figure 12 Exponential beam profiles considered for varying $k$ (see online version for colours)

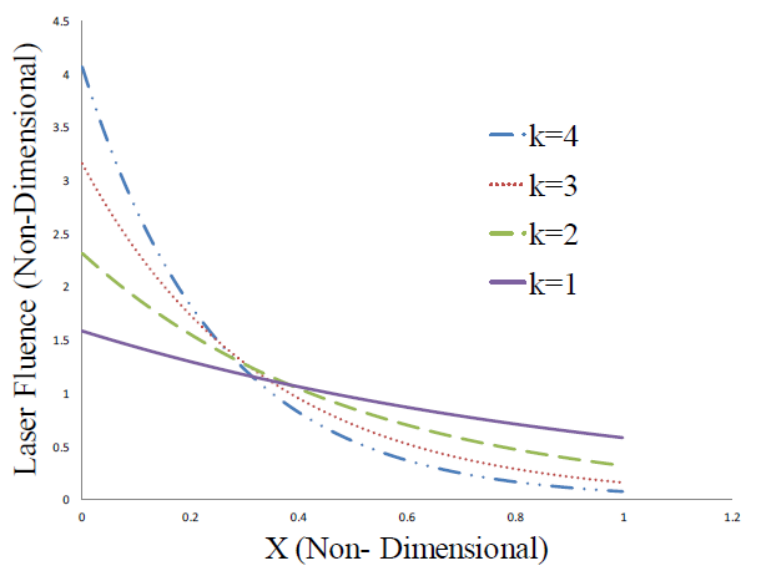

Figure 13 Simulated target profiles for curved surface ablation for exponential profiles with, (a) $k=4$ (b) $k=3$ (c) $k=2$ (d) $k=1$ (see online version for colours)

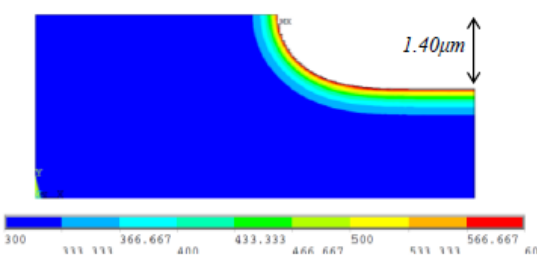

(a)

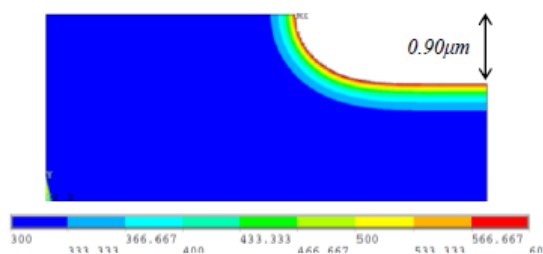

(c)

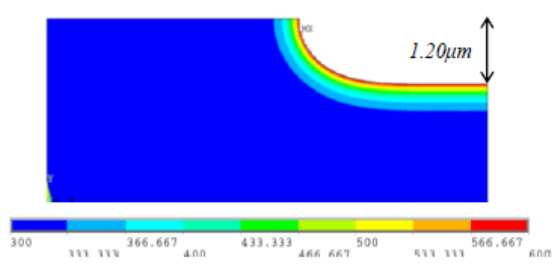

(b)



(d)

\section{Conclusions}

The work presents simulation- and experimental-based study of the curved surface ablation using beam path approach. The approach is based on modifying the laser intensity along the beam cross-section using a mask with spatially varying opacity. A finite element-based numerical model was developed to study the effect of laser intensity profile on the ablated surface profile. The model was validated by comparing the simulated ablation depth with those of the experiments for uniform laser beam profiles. The simulated results of the ablation depth were found to be fairly accurate at low laser fluences in the range of $0.5-1.5 \mathrm{~J} / \mathrm{cm}^{2}$. The modelling approach was extended to simulate the surface profiles of ablated surface by considering spatially varying laser intensity in 
the model. The surface profiles of the ablated surfaces at four different laser intensity profiles viz. flat, triangular, Gaussian and exponential were simulated. It was observed that the surface profiles significantly varied with the laser intensity profiles. For the case of uniform intensity profile, the surface obtained was also nearly flat, except at the edges. However, curved surfaces were obtained for all other cases of laser intensity profiles with Gaussian profile having obtained the maximum ablation depth. Curved surfaces were also generated experimentally using masks with various Gaussian intensity profiles, and the results were in qualitative agreements with the simulation results.

\section{References}

Agari, U., Omura, Y. and Nagai, S. (1997) 'Thermal diffusivity and conductivity of PMMA/PC blends', Polymer, Vol. 38, No. 4, pp.801-807.

Borrelli, N.F. (2004) Microoptics Technology: Fabrication and Applications of Lens Arrays and Devices: Optical Science and Engineering, 2nd ed., CRC Press [online] http://gen.lib.rus.ec/book/index.php?md5=EDFF9C9F1DBE25D06530534E2C82331E.

Braun, A. and Zimmer, K. (2002) 'Diffractive gray scale masks for excimer laser ablation', Applied Surface Science, Vol. 186, Nos. 1-4, pp.200-205.

Christopher, A. (2007) 'Microlens array fabrication via microjet printing technologies', Proc. Workshop Opt. Fabrication Technol.

Feidhlim, T.O. and Sheridan, J.T. (2002) 'Photo resist reflow method of microlens production part I: background and experiments', Optik - International Journal for Light and Electron Optics, Vol. 113, No. 9, pp.391-404.

Fujita, T., Nishihara, H. and Koyama, J. (1981) 'Fabrication of micro lenses using electron beam lithography', Optics Letters, Vol. 6, No. 12, pp.613-615.

Hartmann, D.M., Kibar, O. and Esener, S. (2000) 'Characterization of a polymer microlens fabricated by use of the hydrophobic effect', Optics Letters, Vol. 25, No. 13, pp.975-977.

Hayden, C.J. (2003) 'Three-dimensional excimer laser micromachining using greyscale masks', Journal of Micromechanics and Microengineering, Vol. 13, No. 5, pp.599-603.

Lazare, S. and Granier, V. (1989) 'Ultraviolet laser photoablation of polymers: a review and recent results', Laser Chemistry, Vol. 10, No. 1, pp.25-40.

Marla, D., Bhandarkar, U. and Joshi, S. (2011) 'Critical assessment of the issues in the modeling of ablation and plasma expansion processes in the pulsed laser deposition of metals', Journal of Applied Physics, Vol. 109, No. 2, p.021101.

McKenna, C., Walsh, K., Crain, M. and Lake, J. (2010) 'Maskless direct write grayscale lithography for MEMS applications', Micro/Nano Symposium (UGIM), 2010 18th Biennial University/Government/Industry, pp.1-4.

Naessens, K., Daele, P.V. and Baets, R. (2002) 'Laser ablation based technique for flexible fabrication of microlenses in polymer materials', Proceedings of SPIE, pp.124-127.

Shen, X.J., Pan, L. and Lin, L. (2002) 'Microplastic embossing process: experimental and theoretical characterizations', Sensors and Actuators A: Physical, Vols. 97-98, pp.428-433.

Shin, T.K., Ho, J.R. and Cheng, J.W.J. (2004) 'A new approach to polymeric microlens array fabrication using soft replica molding', IEEE Photonics Technology Letters, Vol. 16, No. 9, pp.2078-2080.

Song, Y.M., Xie, Y., Malyarchuk, V., Xiao, J., Jung, I., Choi, K-J., Liu, Z., Park, H., Lu, C., Kim, R-H., Li, R., Crozier, K.B., Huang, Y. and Rogers, J.A. (2013) 'Digital cameras with designs inspired by the arthropod eye', Nature, Vol. 497, No. 7447, pp.95-99. 